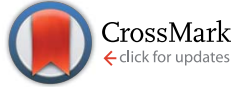

Cite this: RSC Adv., 2015, 5, 42069

\title{
Ordering and dynamics of oligo(phenylene ethynylene) self-assembled monolayers on Au(111)
}

\author{
Hairong Wu, ${ }^{\text {ab }}$ Kai Sotthewes, ${ }^{a}$ Peter M. Schön, ${ }^{b}$ G. Julius Vancso ${ }^{b}$ \\ and Harold J. W. Zandvliet ${ }^{\star a}$
}

\begin{abstract}
Self-assembled monolayers of 4-[4'-(phenylethynyl)-phenylethynyl]-benzenethiolate (PPB-S) molecules on flame annealed $\mathrm{Au}(111)$ have been investigated by ultra-high vacuum scanning tunneling microscopy. We have found a hitherto unknown self-assembled monolayer phase. This phase consists of a finestriped pattern that is aligned along the close packed $\langle 1 \overline{1} 0\rangle$ directions of the Au(111) surface. The finestriped pattern has $\sqrt{ } 3$ periodicity and is separated by narrow vacancy lines which are oriented perpendicular to the fine-striped pattern. Surprisingly, the herringbone reconstruction of the underlying $\mathrm{Au}(111)$ substrate is not fully lifted by the self-assembled monolayer. Spatially resolved current-time scanning tunneling microscopy experiments revealed that the PPB-S molecules at the edges of the vacancy lines exhibit dynamic behavior and frequently jump back and forth between neighboring stripes.
\end{abstract}

Received 27th February 2015 Accepted 27th April 2015

DOI: 10.1039/c5ra03577e

www.rsc.org/advances 4-[4'-(phenylethynyl)-phenylethynyl]-benzenethiol (hereafter referred as PPB-S). The structural and electronic properties of PPB-S have been extensively investigated by scanning tunneling microscopy (STM). ${ }^{22,25-33}$ In two previous studies structural models for the PPB-S SAM have been proposed..$^{\mathbf{3 0}, 31}$ Both articles reported the formation of densely packed SAMs, where the molecules are ordered in several rotational invariant domains. ${ }^{30,31}$ Dhirani et al. used STM to resolve the structure of the PPB-S SAM on Au(111). ${ }^{30}$ These authors found a unit cell with lattice vectors of $a=5.2 \pm 0.4 \AA$ and $b=9.7 \pm 0.9 \AA$, respectively. The angle between both lattice vectors was $\gamma=55^{\circ}$ $\pm 5^{\circ}$. Dhirani et al. pointed out that this unit cell is very close to a $(2 \sqrt{ } 3 \times \sqrt{ } 3) \mathrm{R} 30^{\circ}$ periodicity. In a later study Yang et al. showed that there are six different domains and these authors proposed a $\left(\begin{array}{cc}9 & 4 \\ -2 & 2\end{array}\right)$ unit cell (in terms of the lattice vectors of the underlying $\mathrm{Au}(111)$ substrate). ${ }^{31}$ The measured domain orientations deviate from the hexagonal symmetry of the $\mathrm{Au}(111)$ substrate, suggesting that there is no simple commensurate relationship between the lattices of the SAM and Au(111) substrate.

In this paper, we present a high resolution STM study of PPBS SAMs on $\mathrm{Au}(111)$ substrates. We found that the PPB-S molecules arrange themselves in a hitherto unknown striped phase. Due to an additional substrate treatment step the packing density of this novel phase is slightly lower than the packing density of the already known SAM phases. ${ }^{31}$ The striped pattern is composed of fine stripes and vacancy lines that are oriented perpendicular to these fine stripes. The fine stripes consist on the average of 5 standing up PPB-S molecules, with the actual number fluctuating between 4-6 as the PPB-S molecules or PPB$\mathrm{S}$ molecule complexes at both ends of the fine stripe jump
${ }^{a}$ Physics of Interfaces and Nanomaterials, MESA+ Institute for Nanotechnology, University of Twente, P.O. Box 217, 7500 AE Enschede, The Netherlands. E-mail: h.j. w.zandvliet@utwente.nl

${ }^{b}$ Materials Science and Technology of Polymers, MESA+ Institute for Nanotechnology, University of Twente, P.O. Box 217, 7500 AE Enschede, The Netherlands 
frequently back and forth between fine stripes in neighboring stripes. The surface unit cell involves two fine stripes and exhibits $\sqrt{ } 3 a$ periodicity, where $a$ is the nearest-neighbor distance between the $\mathrm{Au}$ atoms.

\section{Experimental}

Au substrates $\left(11 \times 11 \mathrm{~mm}^{2}, 250 \mathrm{~nm}\right.$ Au on $2 \mathrm{~nm}$ Cr on borosilicate glass) were purchased from Arrandee (Werther, Germany). $\mathrm{Au}(111)$ samples were obtained by annealing these substrates in a high purity $\mathrm{H}_{2}$ flame for $5-10$ minutes. ${ }^{34}$ After flame annealing the $\mathrm{Au}$ substrates were cleaned in a piranha solution $(7: 3$ $\mathrm{H}_{2} \mathrm{SO}_{4}: \mathrm{H}_{2} \mathrm{O}_{2}(30 \%)$ by volume), followed by rinsing with Milli-Q water and ethanol and dried in a nitrogen stream.

$S$-[4-[2-[4-(2-Phenylethynyl)phenyl]ethynyl]phenyl] thioacetate (PPB-SAc, see Fig. 1) was purchased from Sigma-Aldrich and further purified by preparative thin-layer chromatography in dichloromethane-hexane (1:1). Solutions of $50 \mu \mathrm{M}$ of a PPB-SAc were prepared in freshly distilled, deoxygenated tetrahydrofuran (THF) to which $5-10 \mu \mathrm{L}$ of a $25 \%$ aqueous solution of ammonium hydroxide was added. The acetyl groups of PPB-SAc are removed during self-assembly by adding small amount of ammonia. This de-protection activates the thiolate end groups, which then react in situ with the $\mathrm{Au}(111)$ surface and form a selfassembled monolayer. ${ }^{25}$ The thioacetate precursor was used instead of the free thiol to prevent side reactions with functional groups in the SAM precursor backbone.

Subsequently $\mathrm{Au}(111)$ substrates were placed in the PPB-SAc/ THF solution for $\sim 1$ min allowing the conjugated molecules to assemble on the Au surface. After the modification, the substrate was annealed at $40{ }^{\circ} \mathrm{C}$ for 5 hours in ethanol solvent to remove any physisorbed and weakly bound molecules from the surface of the substrate. Finally the substrate was rinsed with pure ethanol and dried with $\mathrm{N}_{2}$ gas. The sample preparation was performed under oxygen-poor conditions to prevent disulfide formation of the PPB-S molecules. The SAMs were immediately inserted into ultra-high vacuum (UHV) for STM measurements.

All STM measurements were performed with an UHV STM (RHK Technology, Inc.) with a base pressure of $1 \times 10^{-10} \mathrm{mbar}$ at room temperature. STM tips were prepared from tungsten wire using electrochemical etching. ${ }^{35}$ For current-time spectroscopy measurements, the tunneling current was recorded as a function of time (I-t traces), while the feedback loop was switched off. Sampling frequencies in the range of $1-100 \mathrm{kHz}$ were used.

Before the formation of the self-assembled monolayer on the $\mathrm{Au}(111)$ substrate we recorded STM images of the bare flame annealed substrate. In Fig. 2a a typical STM image of the bare $\mathrm{Au}(111)$ substrate is shown. The $\mathrm{Au}(111)$ surface exhibits the

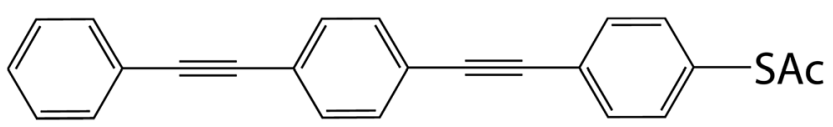

Fig. 1 Chemical structure of PPB-SAc.

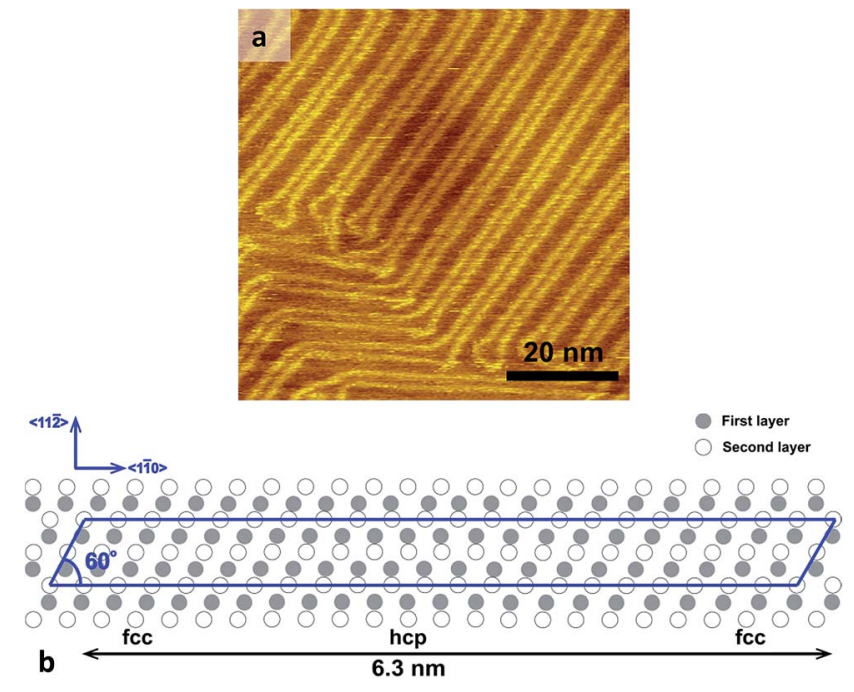

Fig. 2 (a) STM image of bare $A$ (111) with herringbone reconstruction pattern, setpoints are $200 \mathrm{mV}$ and $300 \mathrm{pA}$. (b) Schematic of the herringbone reconstruction. Blue parallelogram indicates the $(22 \times$ $\sqrt{ } 3)$ unit cell.

characteristic herringbone reconstruction. This herringbone reconstruction has a $(22 \times \sqrt{ } 3)$ unit cell where 23 atoms of the top layer are placed on 22 atoms of the second layer (see Fig. $2 \mathrm{~b}$ ). ${ }^{36}$ Along the direction of the compression, the stacking sequence changes from $f c c$ to bridge to $h c p$ and again followed by a bridge, with a periodicity of $63 \AA^{36}{ }^{36}$ The height difference between the $f c c$ domains and the $h c p$ domains is only $15 \mathrm{pm}$. We have used the $\mathrm{Au}(111)$ herringbone reconstruction and the height of the step edges to check the calibration of our Piezo scanner.

\section{Results and discussions}

In contrast to the earlier reported densely packed PPB-S SAM phases on $\mathrm{Au}(111),{ }^{30,31}$ we have found a striped phase. We want to emphasize here that we have obtained this result for three

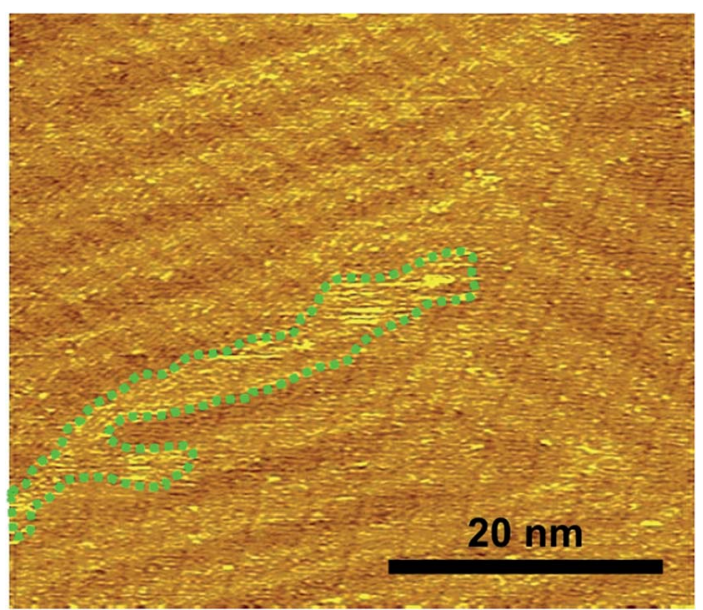

Fig. 3 STM image of PPB-S SAM on Au(111). Image size $50 \times 43.4 \mathrm{~nm}^{2}$. The tunneling parameters are $191 \mathrm{pA}$ and $1.0 \mathrm{~V}$. 
different samples. As is shown in Fig. 3, the herringbone reconstruction pattern underneath the striped phase of the SAMs is still faintly visible, indicating that the formation of this SAM does not completely lift the $\mathrm{Au}(111)$ herringbone reconstruction. It should be pointed out here that the densely packed PPB-S SAM phases reported in ref. 30 and 31 lift the herringbone reconstruction of the underlying $\mathrm{Au}(111)$ substrate. Fig. 4a shows a large scale STM image of the PPB-S SAM. There are three aspects that we want to highlight here. Firstly, high quality layers can be observed; i.e., the molecules pack densely and exhibit perfect crystalline packing over large areas, albeit the packing density is not as high as reported in ref. 30 and 31 . Secondly, the individual fine stripes of the striped phase are well-resolved. It is clear that the PPB-S SAM exhibits a long range ordering. In addition, the vacancy lines between the adjacent striped domains are not perfectly straight, but wander a little bit. Thirdly, the STM images shown in Fig. 3 and $4 \mathrm{a}$ reveal an angle of $30^{\circ}$ for orientation of the fine stripes (indicated by red arrow in Fig. 4a) with respect to high ridges of the $\mathrm{Au}$ herringbone reconstruction (indicated by blue arrow in Fig. 4a); while the angle between the herringbone reconstruction and the broad stripes (indicated by the purple arrow in Fig. 4a) is $60^{\circ}$. In addition, the fine stripes are always out-ofphase with respect to their neighboring fine stripes at the other side of the vacancy line. Interestingly, Noh et al. recently found that the densely packed $c(4 \times 2)$ phase of octanethiol SAM eventually converts to a striped $(6 \times \sqrt{ } 3)$ phase that is very comparable to the striped phase that we found for the PPB-S SAM. ${ }^{37}$ Fig. $4 \mathrm{c}$ and d display the line scans shown in Fig. $4 \mathrm{~b}$ along lines of $\mathrm{A}$ and $\mathrm{B}$, respectively. The spacing between the vacancy lines is $3.46 \pm 0.05 \mathrm{~nm}$, which is approximately 12 times the $\mathrm{Au}(111)$ lattice constant. It should be pointed out here that slightly different vacancy line separations are found as well. The line scan in Fig. 4c reveals that the apparent depth of the trough between the broad stripes is only $\sim 20 \mathrm{pm}$. We should emphasize here that the actual depth of the troughs is much deeper. The vacancy lines are so narrow that the tip of the scanning tunneling microscope is unable to probe the bottom of the troughs. The stripes are composed of fine stripes which aligned in a direction perpendicular to the vacancy lines (see Fig. 4b). The separation between these fine stripes (see Fig. 4d) is $0.50 \pm$ $0.02 \mathrm{~nm}$. As is shown in Fig. 3, the domain boundary (indicated by the area outlined by the dashed green line in Fig. 3) is disordered domain of PPB-S molecules. The vacancy lines (see Fig. 4a and b) exhibit a frizzy appearance, indicating the presence of dynamics.

$I-V$ measurements were performed by the standard method of switching off the STM feedback loop during the time of the bias voltage sweep. A representative current-voltage spectrum of PPB-S SAM on Au(111) is shown in Fig. 5, for a voltage range of $1.5 \mathrm{~V}$ to $-1.5 \mathrm{~V}$. As we can see from Fig. 5, a metallic characteristic $I-V$ curve is observed, implying transport through a conductive medium, which in our case is the conjugated PPB-S molecule.

Next we propose a unit cell of the SAMs based on our high resolution STM images. We refer to these unit cells in units of $a$ $=0.288 \mathrm{~nm}$, which is the nearest-neighbor distance of bulk $\mathrm{Au}$ atoms. Fig. 6a shows the proposed sulfur lattice and the phenyl
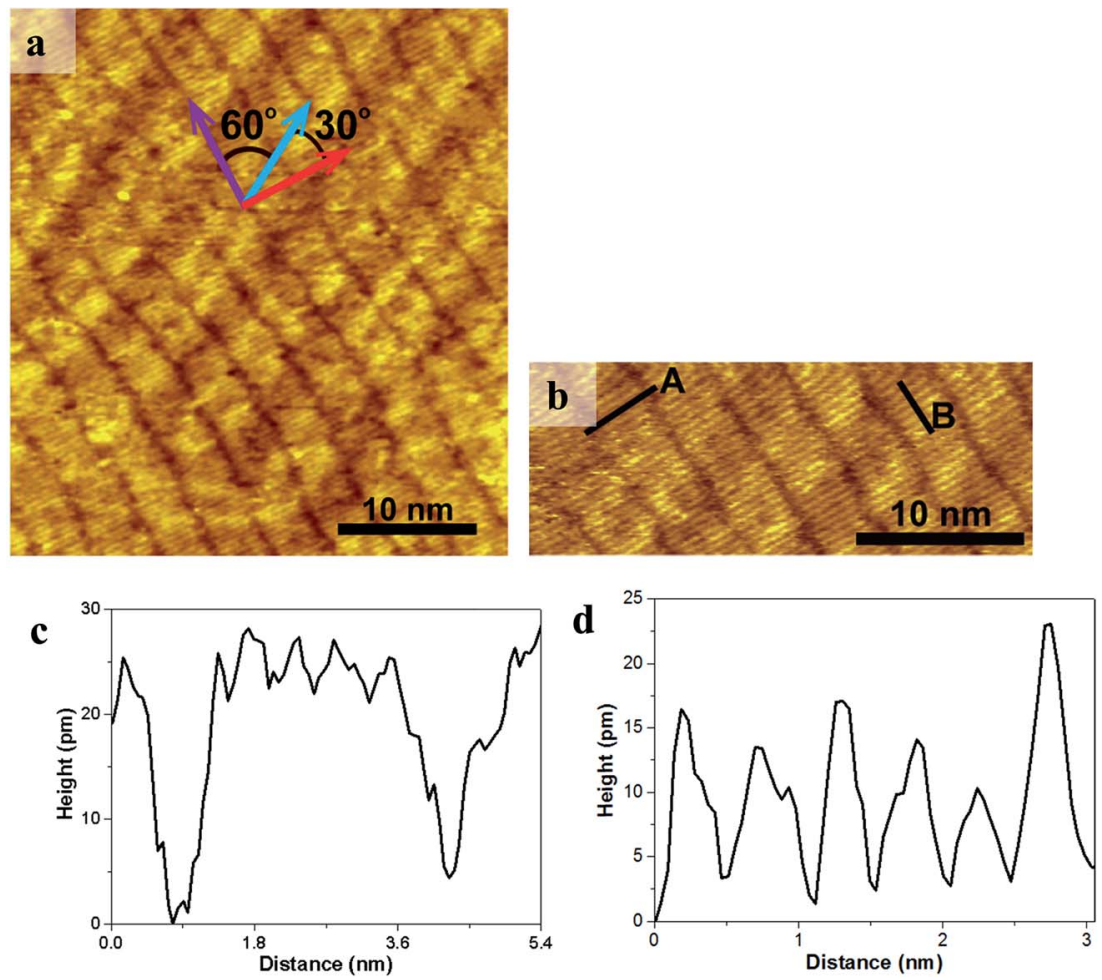

Fig. 4 (a) and (b) STM images of PPB-S SAM on Au(111). Image size is $36.0 \times 35.5 \mathrm{~nm}^{2}$ (a) and $30.0 \times 10.3 \mathrm{~nm}^{2}$ (b). The tunneling parameters are $191 \mathrm{pA}$ and $1.0 \mathrm{~V}$. (c) and (d) line scans shown in (b) along lines of A and B, respectively. 


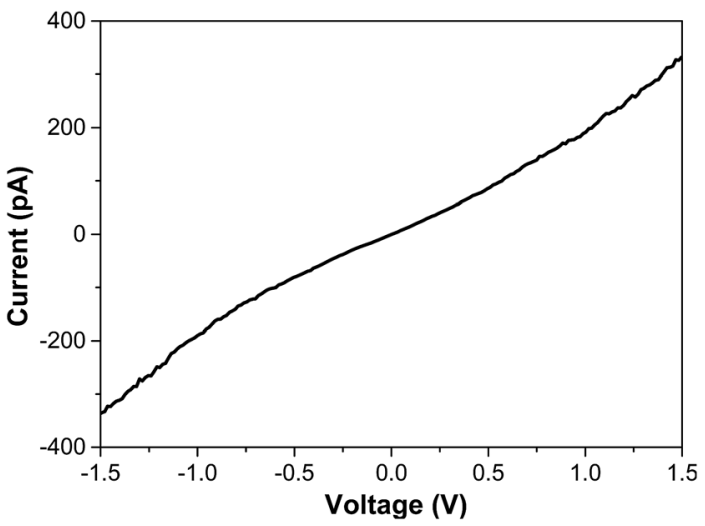

Fig. 5 Current-voltage spectroscopy of PPB-S SAM on Au(111).

ring lattice for SAMs of PPB-S on $\mathrm{Au}(111)$. The blue oval represents the top view of the phenyl ring lattices of PPB-S, while the orange circles represent sulfur lattices. The size of the phenyl ring, which has van der Waals dimensions of $6.4 \AA \times 3.3 \AA$, is substantially larger than that of a sulfur atom. ${ }^{38}$ Considering this size difference between the $\mathrm{S}$ atom and the phenyl ring, it is very likely that the phenyl ring lattice will experience some lateral compressive strain. We believe this strain causes the sulfur atoms to skip a row in order to release the strain at welldefined separations of about 3.0-3.5 nm, resulting in row defects, i.e. vacancy lines, between the striped domains (Fig. 6).
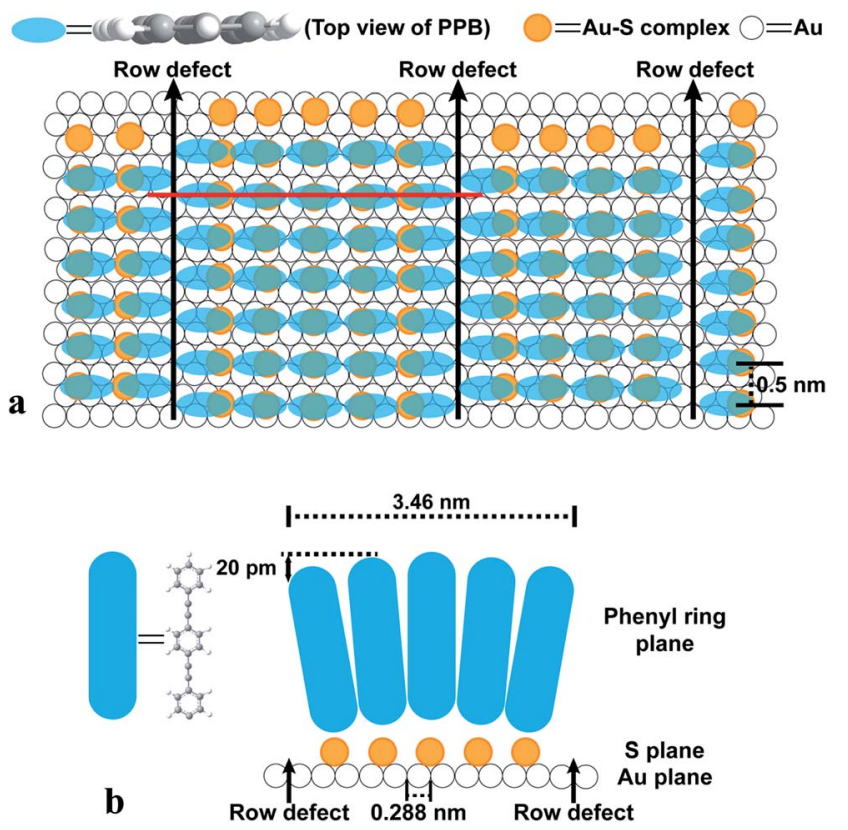

Fig. 6 (a) The proposed model for SAMs of PPB-S: an example in which each fine stripes contains 4 or 5 molecules. The blue oval represents the top view of the phenyl ring lattices of PPB-S, whilst the orange circles represent sulfur atoms. Regarding the molecular sketch, the grey circles refer to the carbon atoms and the white circles to the hydrogen atoms. (b) Side view (red line in figure a) of the phenyl ring plane and the S plane. The blue rod shape stands for the PPB-S molecule lying parallel to the surface.
We would like to emphasize here that the actual bonding of the $\mathrm{S}$ atoms to the $\mathrm{Au}(111)$ is more complex than shown in our schematic diagram. By performing low temperature STM experiments and density functional theory calculations, Maksymovych, Sorescu and Yates Jr. ${ }^{39}$ demonstrated in a convincing way that alkanethiolate species bind to Au adatoms rather than to the regular $\mathrm{Au}$ atoms of the $\mathrm{Au}(111)$ substrate. The $\mathrm{Au}$ adatoms are most probably provided by the pre-existing step edges. Bearing this in mind it is very plausible that also PPB-S molecules bind to $\mathrm{Au}$ adatoms. The occurrence of these PPB-S-Au adatom complexes might also explain why the herringbone reconstruction of the underlying $\mathrm{Au}(111)$ surface is not fully lifted. For the sake of simplicity, however, we assume that the $\mathrm{S}$ atoms are positioned at threefold hollow sites, see Fig. 6a (density functional theory calculations have shown that the Au adatom is not exactly located at a hollow site, but slightly shifted towards a bridge site ${ }^{40-42}$. Considering the diameter of the phenyl ring, the PPB-S molecules align themselves in such a fashion that they are standing upright. We would like to emphasize here that we are not able to extract from our STM data the exact orientation or angle of the long axis of the molecule with respect to the normal of the $\mathrm{Au}(111)$ substrate.

We propose that the phenyl rings of the PPB-S molecules within in a fine stripe are aligned in a plane as depicted in Fig. 6b. The phenyl rings of the PPB-S molecules in a stripe are therefore parallel to the phenyl rings of the PPB-S molecules in the adjacent stripes. In this way intermolecular interactions, such as the $\pi-\pi$ interactions, can stabilize the fine-stripes of the PPB-S SAM on the Au(111) surface. ${ }^{\mathbf{4 3 , 4 4}}$ The effective molecular area per PPB-S molecule in the stripe phase is $\sim 35 \AA^{2}$.

Our model fits the STM images very well. Firstly, the angle between the herringbone pattern and the fine stripes agrees with the observations. As shown in the STM images, the herringbone reconstruction is aligned along the high symmetry direction of the $\mathrm{Au}(111)$ surface. The angle between the fine stripes (orientation indicated by the red arrow in Fig. 4a) and the herringbone reconstruction pattern (orientation indicated by the blue arrow in Fig. 4 a) is $30^{\circ}$. Secondly, both the width of the broad stripes and the spacing between the adjacent fine stripes agree with the measured values. In addition, as displayed in Fig. 6a, the fine stripes are out-of-phase with respect to their neighbors locating at the other side of the vacancy line.

As shown in the STM images, some regions exhibit a frizzy appearance. These frizzy features hint to the occurrence of dynamic events during imaging and mainly occur at the edges of the vacancy lines. In order to study the dynamics, currenttime (denoted $I-t$ hereafter) spectroscopy is performed on various locations on the surface. The $I-t$ traces are recorded with the feedback loop of the STM electronics disabled resulting in a temporal resolution of about $5-10 \mu \mathrm{s}^{45}$ This spectroscopic mode allows us to investigate relatively fast dynamic processes at any predefined position of the surface. Fig. 7 shows a typical example of a $I-t$ trace recorded at a vacancy line. The current flips back and forth between two welldefined levels. The relative current change is substantial, suggesting that a major change takes place at the edges of the vacancy lines. Since the dynamics is only found at the edges of 


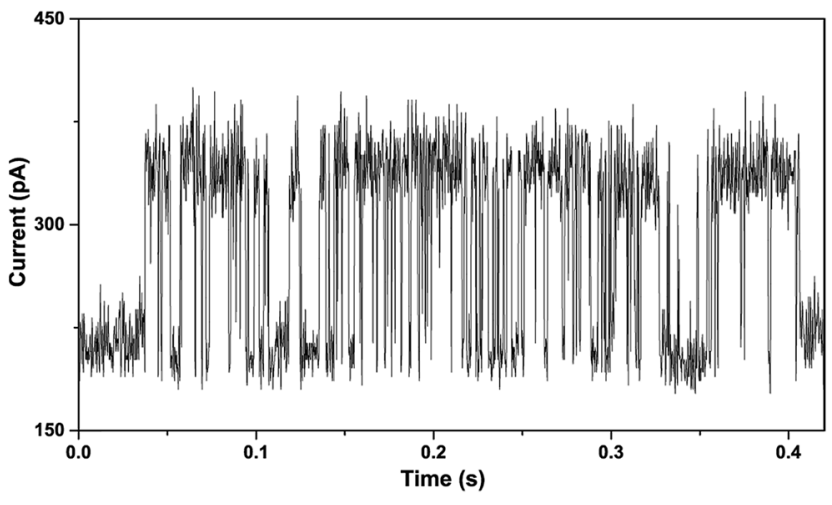

Fig. 7 An exemplary example of $I-t$ trace recorded on the dim line of an PPB-S SAM on Au(111). The set points are 200 pA and $1 \mathrm{~V}$.

the vacancy lines, we propose that PPB-S molecules locating at the edges of the fine stripes jump back and forth between adjacent stripes. Since the sulfur atom of the PPB-S molecule is most probably bound to an Au adatom, the energy barrier for these rearrangement events is rather low. The latter point combined with the observation that the herringbone structure of the underlying $\mathrm{Au}(111)$ surface remains intact provides strong indication in favor of the model that the sulfur atom binds to an $\mathrm{Au}$ adatom rather than to an regular Au surface atom as originally proposed by Maksymovych, Sorescu and Yates Jr. ${ }^{39}$ Interestingly, we recently found a two-level switching behavior for a decanethiol self-assembled monolayer that has a packing density which is somewhat lower than that of the most densely packed standing up phase. ${ }^{46}$

\section{Conclusions}

SAMs of PPB-S on Au(111) surfaces are investigated in ultrahigh vacuum by STM. The SAM is prepared by immersion in a solution followed by annealing at $40{ }^{\circ} \mathrm{C}$ for 5 hours in a pure solvent. By applying this annealing step, a very stable striped phase composed of standing up PPB-S molecules is formed. The width of the stripes is $3.0-3.5 \mathrm{~nm}$, while the distance between the adjacent fine stripes is $0.50 \pm 0.02 \mathrm{~nm}$. A tentative model for the striped phase is proposed in which each fine stripe consists of 4-6 upright standing PPB-S molecules. I- $t$ traces recorded at the edges of the vacancy lines reveal a twolevel switching process, indicative for dynamics of the PPB-S molecules located at the edges of the vacancy lines. It is very likely that sulfur atoms of the PPB-S molecule form a complex with an $\mathrm{Au}$ adatom.

\section{Acknowledgements}

We would like to thank the Stichting voor Fundamenteel Onderzoek der Materie (FOM, 11PR2900) and the Strategic Research Orientation 'Enabling Technologies' of the MESA+ Institute for Nanotechnology for financial support. The authors thank Dr Alejandro Méndez-Ardoy for the purification of the PPB-SAc molecules.

\section{References}

1 A. Riposan, Y. Li, Y. H. Tan, G. Galli and G.-y. Liu, J. Phys. Chem. A, 2007, 111, 12727.

2 D. Wang, L.-J. Wan and C.-L. Bai, Mater. Sci. Eng., R, 2010, 70, 169.

3 Q. K. Ong, S. Zhao, J. Reguera, F. Biscarini and F. Stellacci, Chem. Commun., 2014, 50, 10456.

4 X. Xiao, Y. Wu, M. Sun, J. Zhou, Z. Bo, L. Li and C. Chan, J. Polym. Sci., Part A: Polym. Chem., 2008, 46, 574.

5 W.-f. Dong, R. Wang, G. Mao and H. Möhwald, Soft Matter, 2006, 2, 686.

6 Z. Bo, L. Zhang, B. Zhao, X. Zhang, J. Shen, S. Höppener, L. Chi and H. Fuchs, Chem. Lett., 1998, 27, 1197.

7 J. Mehlich and B. J. Ravoo, Org. Biomol. Chem., 2011, 9, 4108. 8 P. Maksymovych, O. Voznyy, D. B. Dougherty, D. C. Sorescu and J. T. Yates Jr, Prog. Surf. Sci., 2010, 85, 206.

9 D. Kockmann, B. Poelsema and H. J. W. Zandvliet, Nano Lett., 2009, 9, 1147.

10 N. J. Tao, Nat. Nanotechnol., 2006, 1, 173.

11 G. E. Poirier, Langmuir, 1997, 13, 2019.

12 R. L. McCarley, D. J. Dunaway and R. J. Willicut, Langmuir, 1993, 9, 2775.

13 H. Wu, K. Sotthewes, A. Kumar, G. J. Vancso, P. M. Schön and H. J. W. Zandvliet, Langmuir, 2013, 29, 2250.

14 K. Sotthewes, H. Wu, A. Kumar, G. J. Vancso, P. M. Schön and H. J. W. Zandvliet, Langmuir, 2013, 29, 3662.

15 F.-R. F. Fan, Y. Yao, L. Cai, L. Cheng, J. M. Tour and A. J. Bard, J. Am. Chem. Soc., 2004, 126, 4035.

16 F.-R. F. Fan, R. Y. Lai, J. Cornil, Y. Karzazi, J.-L. Brédas, L. Cai, L. Cheng, Y. Yao, D. W. Price, S. M. Dirk, J. M. Tour and A. J. Bard, J. Am. Chem. Soc., 2004, 126, 2568.

$17 \mathrm{H}$. Wu, K. Sotthewes, A. Méndez-Ardoy, T. Kudernac, J. Huskens, A. Lenferink, C. Otto, P. M. Schön, G. J. Vancso and H. J. W. Zandvliet, Chem. Phys. Lett., 2014, 614, 45.

18 X. Xiao, L. A. Nagahara, A. M. Rawlett and N. Tao, J. Am. Chem. Soc., 2005, 127, 9235.

19 C.-P. Chen, W.-R. Luo, C.-N. Chen, S.-M. Wu, S. Hsieh, C.-M. Chiang and T.-Y. Dong, Langmuir, 2013, 29, 3106.

20 Q. Lu, K. Liu, H. Zhang, Z. Du, X. Wang and F. Wang, ACS Nano, 2009, 3, 3861.

21 S.-S. Jester, D. Schmitz, F. Eberhagen and S. Höger, Chem. Commun., 2011, 47, 8838.

22 H. Kim, S. S. Jang, R. A. Kiehl and W. A. Goddard, J. Phys. Chem. C, 2011, 115, 3722.

23 J. Cornil, Y. Karzazi and J. L. Brédas, J. Am. Chem. Soc., 2002, 124, 3516.

24 N. Majumdar, N. Gergel-Hackett, J. C. Bean, L. R. Harriott, G. Pattanaik, G. Zangari, Y. Yao and J. M. Tour, J. Electron. Mater., 2006, 35, 140.

25 A.-S. Hallbäck, B. Poelsema and H. J. W. Zandvliet, ChemPhysChem, 2007, 8, 661.

26 Z. J. Donhauser, B. A. Mantooth, K. F. Kelly, L. A. Bumm, J. D. Monnell, J. J. Stapleton, D. W. Price, A. M. Rawlett, D. L. Allara, J. M. Tour and P. S. Weiss, Science, 2001, 292, 2303. 
27 G. K. Ramachandran, T. J. Hopson, A. M. Rawlett, L. A. Nagahara, A. Primak and S. M. Lindsay, Science, 2003, 300, 1413.

28 K. Walzer, E. Marx, N. C. Greenham, R. J. Less, P. R. Raithby and K. Stokbro, J. Am. Chem. Soc., 2004, 126, 1229.

29 W. Li, G. G. Langlois, N. A. Kautz and S. J. Sibener, J. Phys. Chem. C, 2014, 118, 15846.

30 A.-A. Dhirani, R. W. Zehner, R. P. Hsung, P. Guyot-Sionnest and L. R. Sita, J. Am. Chem. Soc., 1996, 118, 3319.

31 G. Yang, Y. Qian, C. Engtrakul, L. R. Sita and G. Liu, J. Phys. Chem. B, 2000, 104, 9059.

32 N. Gergel, N. Majumdar, K. Keyvanfar, N. Swami, L. R. Harriott, J. C. Bean, G. Pattanaik, G. Zangari, Y. Yao and J. M. Tour, J. Vac. Sci. Technol., A, 2005, 23, 880.

33 G. R. Dholakia, W. Fan, J. Koehne, J. Han and M. Meyyappan, J. Nanosci. Nanotechnol., 2003, 3, 231.

34 H. Schönherr, M. W. J. Beulen, J. Bügler, J. Huskens, F. C. J. M. Van Veggel, D. N. Reinhoudt and G. J. Vancso, J. Am. Chem. Soc., 2000, 122, 4963.

35 J. P. Ibe, J. P. P. Bey, S. L. Brandow, R. A. Brizzolara, N. A. Burnham, D. P. DiLella, K. P. Lee, C. R. K. Marrian and R. J. Colton, J. Vac. Sci. Technol., A, 1990, 8, 3570.
36 C. Wöll, S. Chiang, R. J. Wilson and P. H. Lippel, Phys. Rev. B: Condens. Matter Mater. Phys., 1989, 39, 7988.

37 J. Noh, H. S. Kato, M. Kawai and M. Hara, J. Phys. Chem. B, 2006, 110, 2793.

38 G. Yang and G. Liu, J. Phys. Chem. B, 2003, 107, 8746.

39 P. Maksymovych, D. C. Sorescu and J. T. Yates Jr, Phys. Rev. Lett., 2006, 97, 146103.

40 M. C. Vargas, P. Giannozzi, A. Selloni and G. Scoles, J. Phys. Chem. B, 2001, 105, 9509.

41 Y. Yourdshahyan and A. M. Rappe, J. Chem. Phys., 2002, 117, 825.

42 J. Gottschalck and B. Hammer, J. Chem. Phys., 2002, 116, 784. 43 R.-F. Dou, X.-C. Ma, L. Xi, H. L. Yip, K. Y. Wong, W. M. Lau, X.-L. Fan, J.-F. Jia, Q.-K. Xue, W. S. Yang, H. Ma and A. K.-Y. Jen, J. Phys.: Condens. Matter, 2008, 20, 315012.

44 R.-F. Dou, X.-C. Ma, L. Xi, H. L. Yip, K. Y. Wong, W. M. Lau, J.-F. Jia, Q.-K. Xue, W.-S. Yang, H. Ma and A. K. Y. Jen, Langmuir, 2006, 22, 3049.

45 A. Van Houselt and H. J. W. Zandvliet, Rev. Mod. Phys., 2010, 82, 1593.

46 K. Sotthewes, H. Wu and H. J. W. Zandvliet, unpublished. 